\title{
Deep Subwavelength Power Concentration-Based Hyperbolic Metamaterials
}

\author{
Amanpreet Kaur, ${ }^{1}$ Saptarshi Banerjee, ${ }^{1}$ Wangshi Zhao, ${ }^{1}$ \\ Jayanti Venkataraman, ${ }^{2}$ and Zhaolin $\mathrm{Lu}^{1,2}$ \\ ${ }^{1}$ Microsystems Engineering, Kate Gleason College of Engineering, Rochester Institute of Technology, Rochester, NY 14623, USA
${ }^{2}$ Department of Electrical and Microelectronic Engineering, Kate Gleason College of Engineering, Rochester Institute of Technology,
Rochester, NY 14623, USA
}

Correspondence should be addressed to Amanpreet Kaur, axk6400@rit.edu

Received 31 May 2012; Revised 6 August 2012; Accepted 27 August 2012

Academic Editor: Xiaoyue Huang

Copyright ( $) 2012$ Amanpreet Kaur et al. This is an open access article distributed under the Creative Commons Attribution License, which permits unrestricted use, distribution, and reproduction in any medium, provided the original work is properly cited.

\begin{abstract}
Hyperbolic metamaterials can manipulate electromagnetic waves by converting evanescent waves into propagating waves and thus support light propagation without diffraction limit. In this paper, deep subwavelength focusing (or power concentration) is demonstrated both numerically and experimentally using hyperbolic metamaterials. The results verify that hyperbolic metamaterials can focus a broad collimated beam to spot size of $\sim \lambda_{0} / 6$ using wired medium design for both normal and oblique incidence. The nonmagnetic design, no-cut-off operation, and preferred direction of propagation in these materials significantly reduce the attenuation in electromagnetic waves.
\end{abstract}

In conventional materials, focusing is diffraction limited due to the loss of evanescent waves which carry subwavelength information. Therefore, projecting a subdiffraction-limited image would require the recovery of the evanescent waves. The negative index material (NIM), which simultaneously has negative permittivity and negative permeability, was first theoretically studied by Veselago in 1968 [1]. Many years later, Pendry theoretically investigated that a slab of NIM can be used to make a "perfect lens" [2]. Since then, various designs have been explored, and negative refraction has been demonstrated from microwave to optical frequencies [3-9]. The realization of a lens using NIM in practice is rather difficult as negative values of $\varepsilon$ and $\mu$ are very sensitive to frequency, and ideal condition can only be satisfied at carefully selected frequencies. Some properties of the NIM are based on the resonance of the magnetic component of electromagnetic (EM) wave and thus suffer from energy loss due to the dissipation at the resonances, which reduces the resolution of an imaging system significantly [10-12]. Recently, negative refraction effect and imaging by negative refraction were proposed and demonstrated in indefinite metamaterials [1322]. Indefinite metamaterials are anisotropic material, in which one or more components of the permittivity tensor have negative sign while others are positive, which results in hyperbolic dispersion relation. These materials are therefore also called hyperbolic metamaterials (HMMs). In a twodimensional imaging system (assuming that the material is nonmagnetic), the dispersion relation can be expressed as

$$
\frac{k_{z}^{2}}{\varepsilon_{x}}+\frac{k_{x}^{2}}{\varepsilon_{z}}=k_{0}^{2}=\frac{\omega^{2}}{c^{2}}, \quad\left(\varepsilon_{x}>0, \varepsilon_{z}<0\right),
$$

where $\omega$ is angular frequency, and $c$ is the speed of light in free space while $k_{x}, k_{z}$, and $k_{0}$ are the wavenumbers (or spatial frequencies) in the transverse direction $x$, propagation direction $z$, and free space, respectively. Compared with the circular dispersion relation in a conventional isotropic material (Figure 1(a)) and elliptical relation (Figure 1(b)) in anisotropic material with finite permittivity $\left(\varepsilon_{x}, \varepsilon_{z}>0\right)$, the dispersion curve for an indefinite metamaterial $\left(\varepsilon_{x}>0\right.$, $\left.\varepsilon_{z}<0\right)$ is hyperbolic as shown in Figure 1(c). From (1), we have

$$
k_{z}=\sqrt{\varepsilon_{x}\left(k_{0}^{2}-\frac{k_{x}^{2}}{\varepsilon_{z}}\right)}
$$




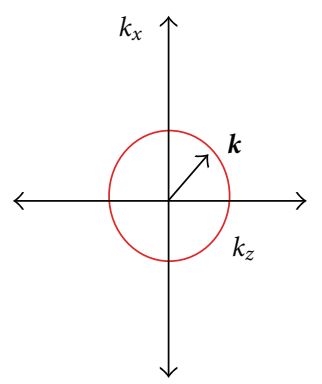

(a)

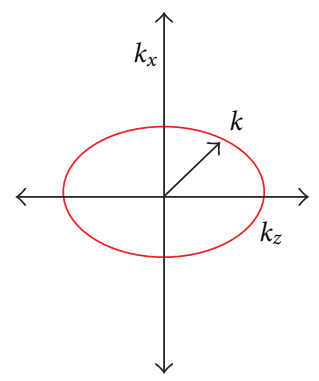

(b)

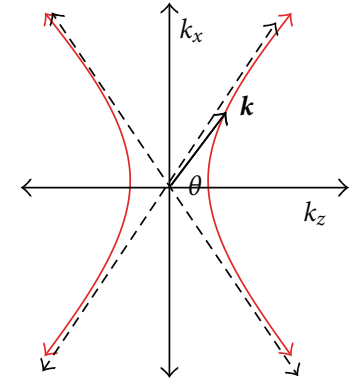

(c)

FIGURE 1: (a) Equifrequency surface diagram for an isotropic medium $\left(\varepsilon_{z}=\varepsilon_{x}\right.$ ). (b) Equifrequency surface diagram for an anisotropic medium $\left(\varepsilon_{z} \neq \varepsilon_{x}\right)$. (c) Equifrequency surface diagram for an indefinite medium (hyperbolic) with $\left(\varepsilon_{z}<0, \varepsilon_{x}>0\right)$.

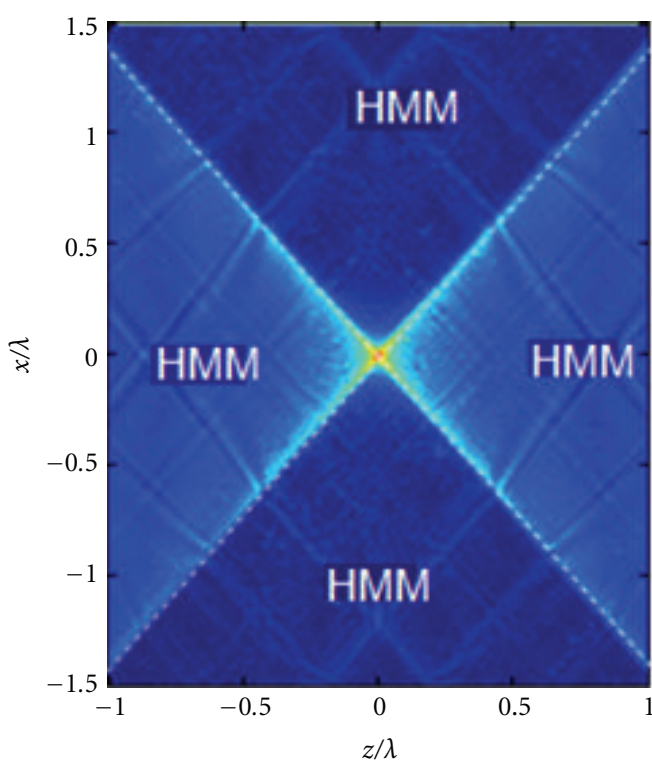

(a)

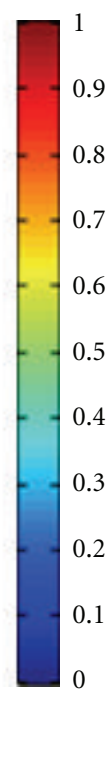

FIGURE 2: (a) Radiation of a point source inside a $\operatorname{HMM}\left(\varepsilon_{z}=-4\right.$ and $\left.\varepsilon_{x}=1\right)$. (b) Light propagation through a sharp edge aperture in HMM and the cross of preferred directions resulting in focusing.

For an elliptically dispersive material there is a theoretical cutoff on the spatial frequency vector while on the other hand there is no limit in dispersion relation of a hyperbolically dispersive material. Assuming that the optical axis is along the $z$ direction, the transfer function for such a system is given by

$$
H\left(k_{x}\right)=\exp \left(j k_{z} z\right)=\exp \left(j z \sqrt{\varepsilon_{x}} \sqrt{k_{0}^{2}-\frac{k_{x}^{2}}{\varepsilon_{z}}}\right) .
$$

When $\varepsilon_{z}<0$ and $\varepsilon_{x}>0$, there is no cutoff for any high spatial frequency $k_{x}$ varying from $-\infty$ to $+\infty$. This means that the HMM can convert the evanescent waves in conventional media into propagating waves. The losses in the HMM also play an important role in the performance as different $k$ components may have different propagation lengths in a HMM; thus the $k$ vectors attenuate differently.

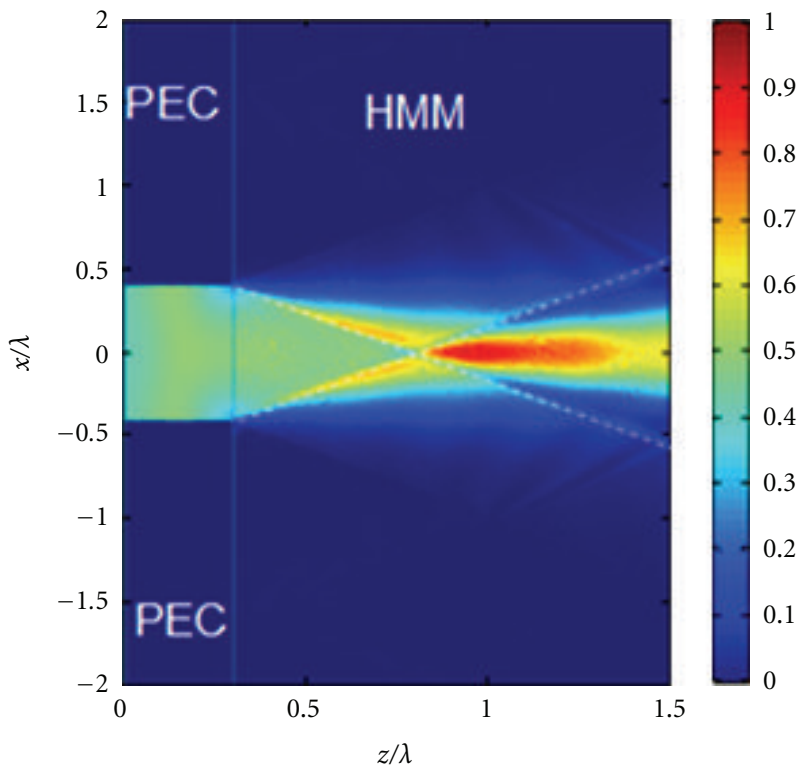

(b) 

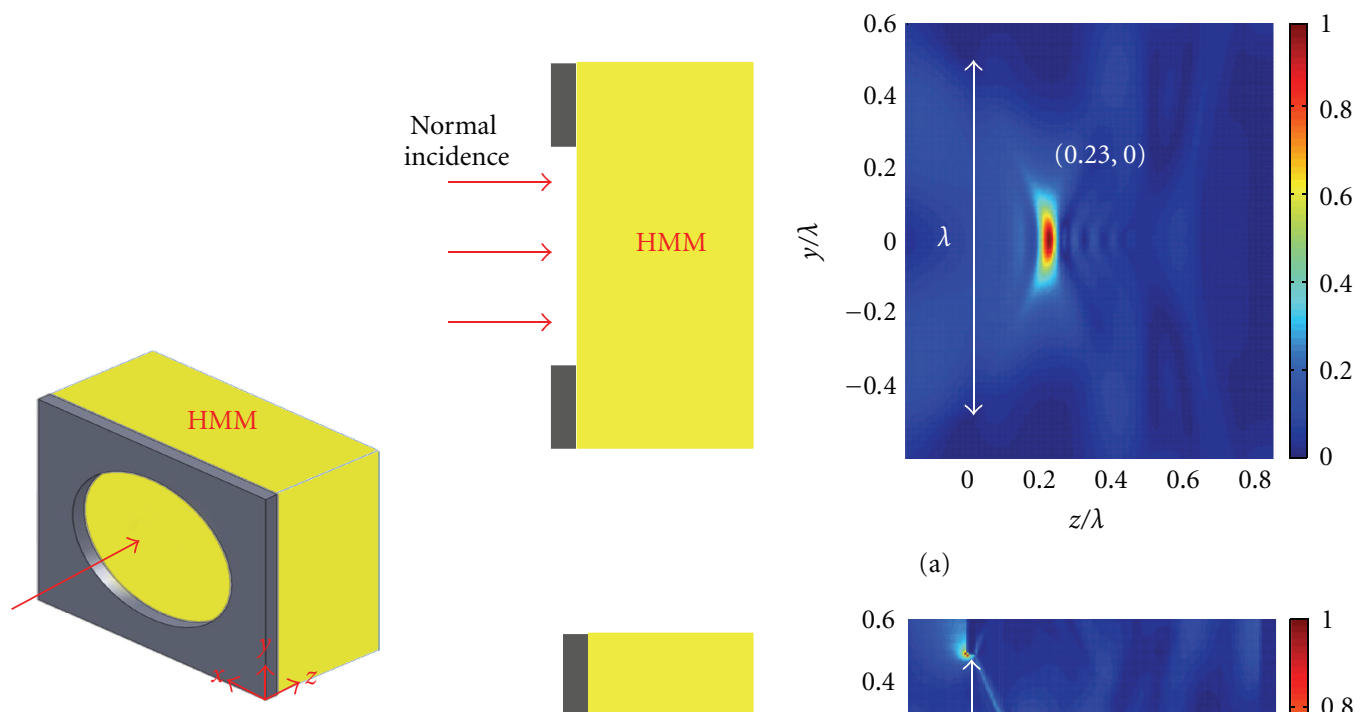

(a)

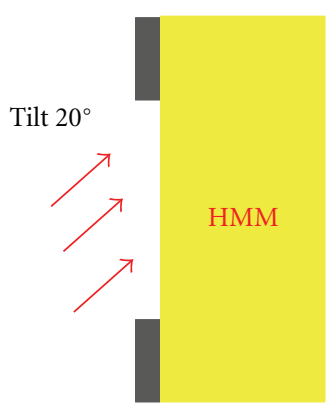

(b)

Figure 3: Numerical simulation results for focusing using HMM (a) at normal incidence (b) with tilt angle of $20^{\circ}$.

used to excite high spatial frequency components. As shown in Figure 2(b), the light from such an aperture propagates in the direction of asymptotes and is focused at the intersection of them. Note that all focused waves in the HMM are propagating waves. This effect can also be extended into threedimensional (3D) configurations. Thus, the propagation of high spatial frequency components and preferred direction gives us confidence to achieve subwavelength focusing using a slab of a HMM. In contrast, negative refractive index material can make flat lenses, but focusing a broad collimated beam into deep subwavelength spot size may require curved surface area of the lens [23].

Another feature of the lens is that the location of the focused spot is not sensitive to the incident angle. To verify the subwavelength focusing at different incidence angles in a HMM slab, some 3D FDTD simulations were performed using the Lumerical FDTD solution software. In simulations, a HMM medium with the effective permittivity $\left(\varepsilon_{z}=-1\right.$, and $\varepsilon_{x}=1$ ) was assumed. The simulations were performed for normal incidence as well as oblique incidence of $20^{\circ}$, and the resulting field distribution is shown in Figures 3(b) and 3 (c), respectively. It clearly verifies focusing using the HMM structure at two different incident angles. In addition, the incident angle of the $20^{\circ}$ focus spot is shifted only by a small distance. The reason is that the location of the focused spot depends on the material properties, or more specifically the ratio of $\varepsilon_{x}\left(\varepsilon_{y}\right)$ and $\varepsilon_{z}$.

In comparison to NIM, HMMs are easier to fabricate as only permittivity in one direction is required to be negative, and it does not rely on magnetic resonance. In addition, most metamaterials are inherently anisotropic $[21,22,24]$. In this paper, we also present experimental demonstration of deep subwavelength focusing using a HMM. A HMM structure can be realized using wired medium design in which an array of parallel conducting wires are embedded in a matrix of a dielectric material as illustrated in Figure 4(a). The wire medium structure exhibits novel electromagnetic properties in the $\mathrm{GHz}$ region. In the wired medium, parallel wires are separated from each other by a certain lattice constant, and, due to this periodic arrangement, the effective plasma frequency $\left(\omega_{p}\right)$ can be derived for the collective oscillation of electrons $[7,25]$ and is given as

$$
\omega_{p}^{2}=\frac{2 \pi c^{2}}{a^{2} \ln (a / r)},
$$

where $a$ is lattice constant and $r$ is radius of the metal wire. For incident waves with parallel polarization, the wired 


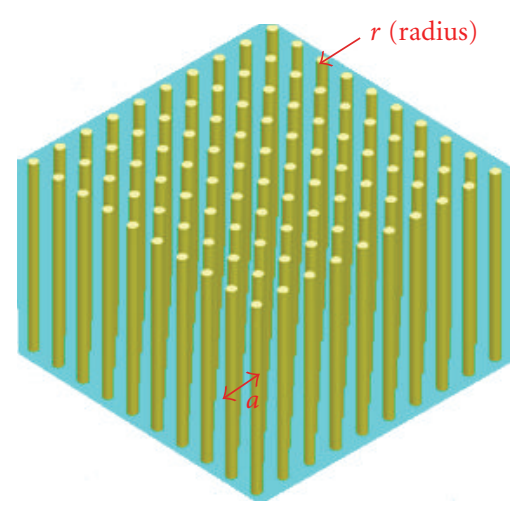

(a)
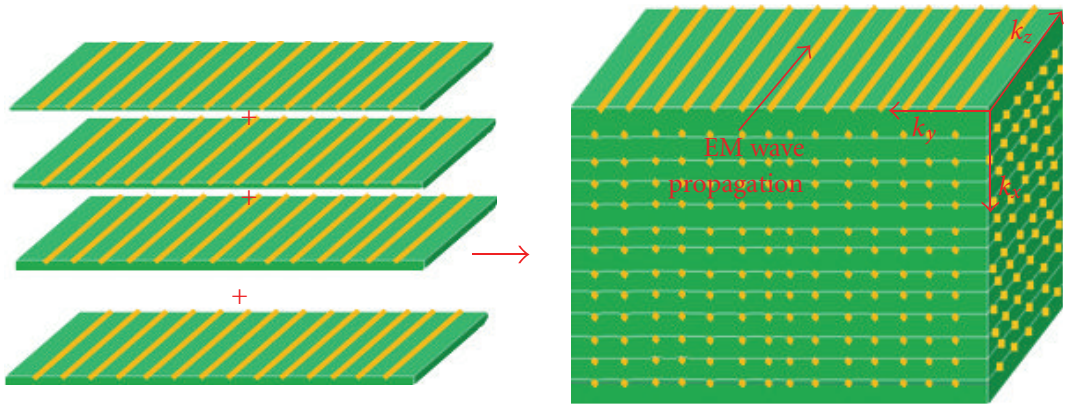

(b)

FIGURE 4: (a) Schematic illustration of a wired medium design. (b) Illustration of the HMM slab structure (yellow-metal wires), green (PCB slab).

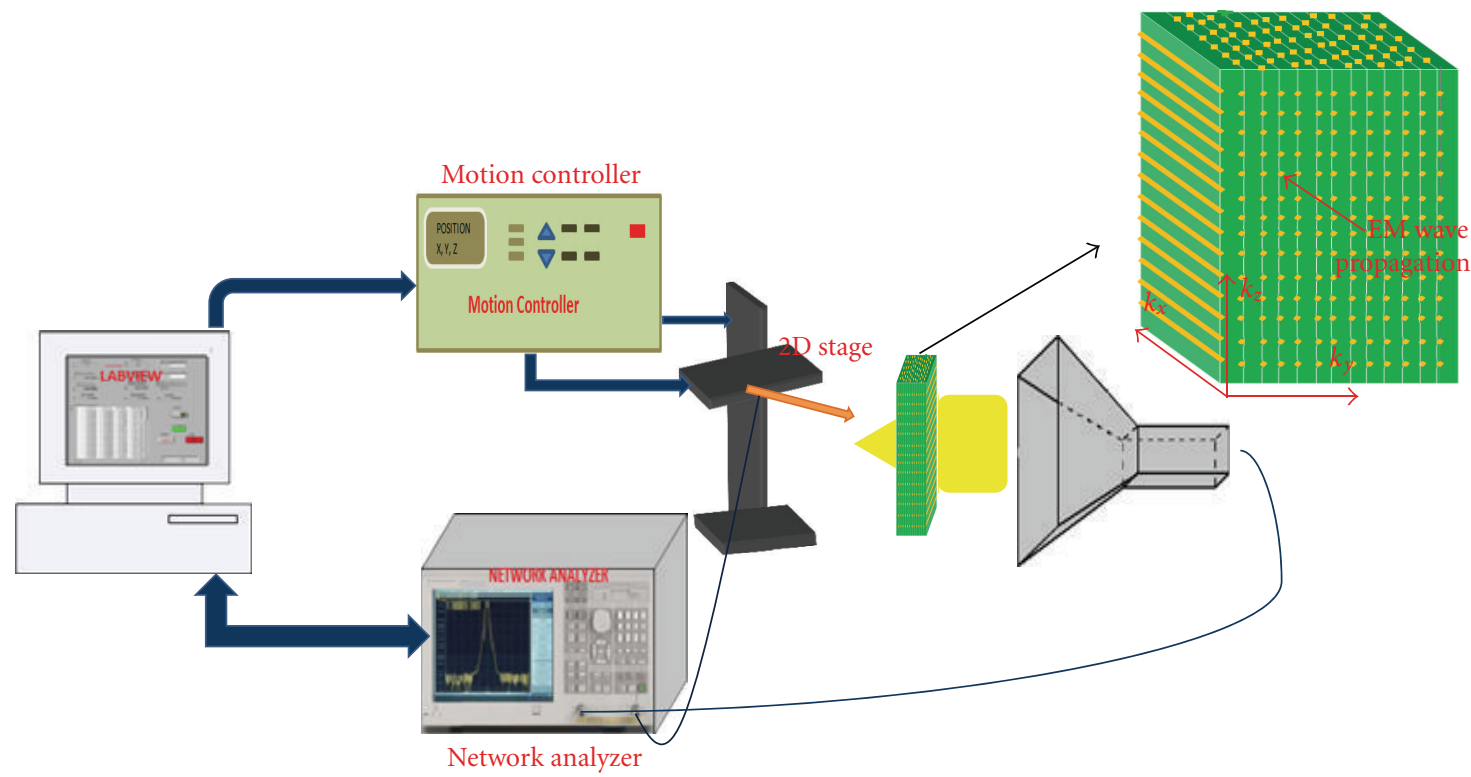

FIGURE 5: Schematic diagram of the experimental setup for superfocusing in the HMM.

medium can be characterized by an effective permittivity $\varepsilon_{z}=1-\left(\omega_{p}^{2} / \omega^{2}\right)$ in the direction of propagation. Below the plasma frequency, dielectric constant $\left(\varepsilon_{z}\right)$ becomes negative, and above the plasma frequency the $\varepsilon_{z}$ becomes positive. However, when the electric field is no longer parallel to the wires, the wire medium exhibits spatial dispersion [25]. The geometric parameters used in the HMM design, that is, the wire radius and lattice constant, are much smaller than the free-space wavelength $\left(\lambda_{0}\right)$ of the incident EM wave. Thus, this medium can be considered as a homogenous effective medium [26]. Printed circuit boards (PCBs) were used as dielectric, and copper was used as metal wires which behave as perfect electric conductor (PEC) at microwave frequencies. Very thin metal wires are embedded periodically on top of a PCB slab of thickness $a$. These copper wires were etched on the top surface of the PCB and run parallel to the breadth of the board. The radius of copper wire used was approximately $0.1 \mathrm{~mm}$, and the resulting lattice constant of the structure was $a=2 \mathrm{~mm}$. The dimensions of a single PCB plate are $12 \mathrm{~cm} \times 2.5 \mathrm{~cm} \times 0.2 \mathrm{~cm}$. Then 65 such slabs were stacked on top of each other to act equivalently to a wired medium structure as shown in Figure 4(b). The permittivity along the direction of propagation $\varepsilon_{z}$ is -37.49 for a plasma frequency of $34.58 \mathrm{GHz}$ which is calculated using (4). In the experimental setup (Figure 5), a collimated beam from a horn antenna is incident on the HMM slab along the direction of metal wires. The antenna was directly fed by a coaxial cable from a vector network analyzer with frequency ranging from 4.0 to $5.8 \mathrm{GHz}$. A circular aperture mask of diameter $80 \mathrm{~mm}$ was made out of aluminum so as to define the input beam and excite high spatial frequency components at the aperture edge. The center position of the slab and the transmitted beam is aligned up to act as the reference under the normal incidence. 


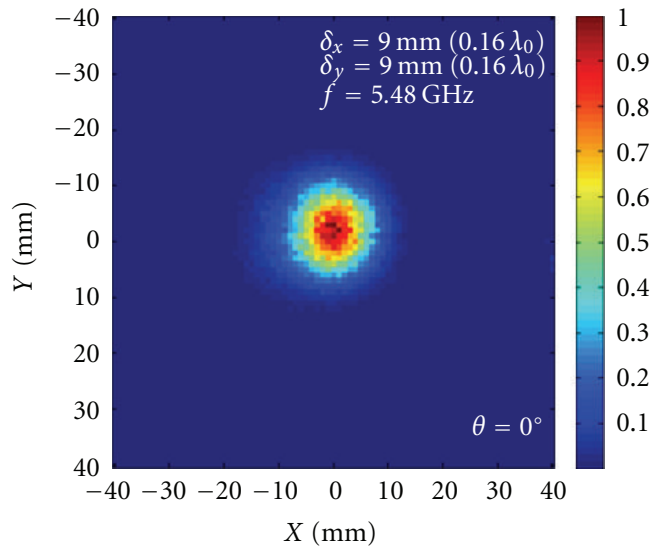

(a)

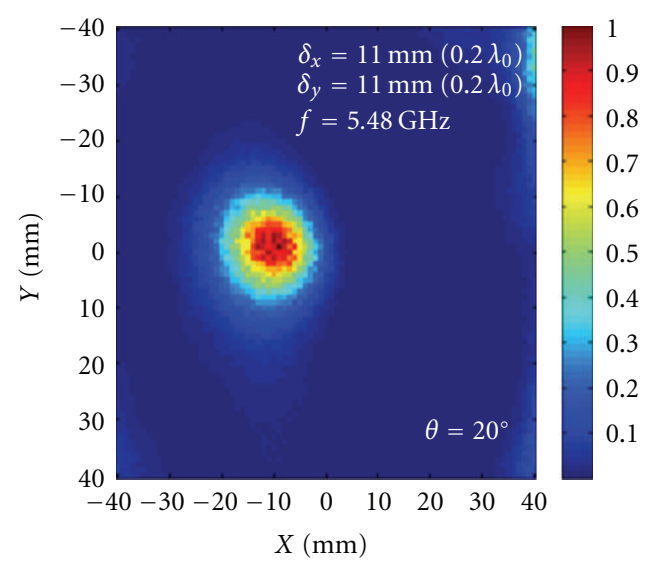

(b)

Figure 6: (a) Experimental verification of focusing a broad collimated $(\Phi 80 \mathrm{~mm})$ beam to a spot size of $9 \mathrm{~mm} \times 9 \mathrm{~mm}\left(\lambda_{0} / 6\right)$ at normal incidence. (b) Focusing to $11 \mathrm{~mm} \times 11 \mathrm{~mm}\left(\lambda_{0} / 5\right)$ at oblique incidence.

The electromagnetic waves transmitted through the slab are mapped by a near-field scanning probe which was controlled by a motion controller via LabVIEW programming. The measurements were done in $x$ and $y$ directions, and the best quality of focusing was observed at $5.48 \mathrm{GHz}$. It was verified that light from a larger apperture size $(\Phi 80 \mathrm{~mm})$ can be focused to deep-subwavelength image size of $9 \times 9 \mathrm{~mm}$ which is approximately $\lambda_{0} / 6$ at normal incidence and $11 \times$ $11 \mathrm{~mm}\left(\lambda_{0} / 5\right)$ at incidence angle of $20^{\circ}$. The experimental results are shown in Figures 6(a) and 6(b). However, we had done experiments at different incident angles that is at $20^{\circ}$, $30^{\circ}$, and $40^{\circ}$ and found that focusing is achieved at all the angles, and the focus will be shifted accordingly. The greater the tilt angle, the greater the shift in focus. If the size of aperture is further reduced, focusing was still observed but with a different focal length. Thus, the wire medium supports the transmission of propagating modes for any angle of incidence.

In conclusion, we reported super focusing (power concentration) using a HMM based on a wired medium design. This focusing was verified both numerically and experimentally at gigahertz frequencies with different angles of incidence. It is experimentally verified that higher spatial frequency components can be excited using a sharp apperture for a broad collimated beam. The subwavelength focusing of $\lambda_{0} / 6$ has been demonstrated. This is possible due to coupling of higher spatial frequency components into the preferred directions of propagation inside a HMM. The present realization of the HMM using wire medium is advantageous at microwave frequencies. This phenomenon can be further explored for applications in nanolithography, optical tweezers, solar cells, nanowaveguide coupler, and optical storage.

\section{Acknowledgments}

Acknowledgment is made to the Donors of the American Chemical Society Petroleum Research Fund for partial support of this research and RIT Research Computing for technical support. The numerical simulation was based on FDTD solutions from Lumerical Solution, Inc. (http:// www.lumerical.com/).

\section{References}

[1] V. G. Veselago, "The electrodynamics of substances with simultaneously negative values of $\varepsilon$ and $\mu$," Soviet Physics Uspekhi, vol. 10, pp. 509-514, 1968.

[2] J. B. Pendry, "Negative refraction makes a perfect lens," Physical Review Letters, vol. 85, no. 18, pp. 3966-3969, 2000.

[3] R. A. Shelby, D. R. Smith, and S. Schultz, "Experimental verification of a negative index of refraction," Science, vol. 292, no. 5514, pp. 77-79, 2001.

[4] A. A. Houck, J. B. Brock, and I. L. Chuang, "Experimental observations of a left-handed material that obeys Snell's law," Physical Review Letters, vol. 90, no. 13, Article ID 137401, 4 pages, 2003.

[5] X. Zhang and Z. Liu, "Superlenses to overcome the diffraction limit," Nature Materials, vol. 7, no. 6, pp. 435-441, 2008.

[6] N. Fang, H. Lee, C. Sun, and X. Zhang, "Sub-diffractionlimited optical imaging with a silver superlens," Science, vol. 308, no. 5721, pp. 534-537, 2005.

[7] J. B. Pendry, A. J. Holden, W. J. Stewart, and I. Youngs, "Extremely low frequency plasmons in metallic mesostructures," Physical Review Letters, vol. 76, no. 25, pp. 4773-4776, 1996.

[8] A. Berrier, M. Mulot, M. Swillo et al., "Negative refraction at infrared wavelengths in a two-dimensional photonic crystal," Physical Review Letters, vol. 93, no. 7, Article ID 073902, 4 pages, 2004.

[9] C. Luo, S. G. Johnson, J. D. Joannopoulos, and J. B. Pendry, "Negative refraction without negative index in metallic photonic crystals," Optics Express, vol. 11, no. 7, pp. 746-754, 2003.

[10] M. Born and E. Wolf, Principles of Optics: Electromagnetic Theory of Propagation, Interference and Diffraction of Light, Cambridge University Press, 7th edition, 1999.

[11] J. O. Dimmock, "Losses in left-handed materials," Optics Express, vol. 11, no. 19, pp. 2397-2402, 2003.

[12] I. A. Larkin and M. I. Stockman, "Imperfect perfect lens," Nano Letters, vol. 5, no. 2, pp. 339-343, 2005. 
[13] D. R. Smith and D. Schurig, "Electromagnetic wave propagation in media with indefinite permittivity and permeability tensors," Physical Review Letters, vol. 90, no. 7, Article ID 077405, 4 pages, 2003.

[14] D. R. Smith, P. Kolinko, and D. Schurig, "Negative refraction in indefinite media," Journal of the Optical Society of America $B$, vol. 21, no. 5, pp. 1032-1043, 2004.

[15] M. Cheng and R. Chen, "Large positive and negative lateral shifts from an anisotropic metamaterial slab backed by a metal," Chinese Physics Letters, vol. 26, no. 1, Article ID 014101, 2009.

[16] W. Yan, L. Shen, L. Ran, and J. A. Kong, "Surface modes at the interfaces between isotropic media and indefinite media," Journal of the Optical Society of America A, vol. 24, no. 2, pp. 530-535, 2007.

[17] L. Zhang, Q. Wang, Y. Zhao, W. Qiao, and J. Zhao, "Surface modes of the sandwhich structure containing indefinite metamaterials," in Proceedings of the International Conference on Microwave and Millimeter Wave Technology (ICMMT '10), pp. 1669-1672, May 2010.

[18] D. R. Smith, D. Schurig, J. J. Mock, P. Kolinko, and P. Rye, "Partial focusing of radiation by a slab of indefinite media," Applied Physics Letters, vol. 84, no. 13, pp. 2244-2246, 2004.

[19] R. Wangberg, J. Elser, E. E. Narimanov, and V. A. Podolskiy, "Nonmagnetic nanocomposites for optical and infrared negative-refractive-index media," Journal of the Optical Society of America B, vol. 23, no. 3, pp. 498-505, 2006.

[20] Z. Jacob, L. V. Alekseyev, and E. Narimanov, "Optical hyperlens: far-field imaging beyond the diffraction limit," Optics Express, vol. 14, no. 18, pp. 8247-8256, 2006.

[21] B. Wood, J. B. Pendry, and D. P. Tsai, "Directed subwavelength imaging using a layered metal-dielectric system," Physical Review B, vol. 74, no. 11, Article ID 115116, 8 pages, 2006.

[22] Y. Liu, G. Bartal, and X. Zhang, "All-angle negative refraction and imaging in a bulk medium made of metallic nanowires in the visible region," Optics Express, vol. 16, no. 20, pp. 1543915448, 2008.

[23] J. B. Pendry, "Perfect cylindrical lenses," Optics Express, vol. 11, no. 7, pp. 755-760, 2003.

[24] W. Cai, D. A. Genov, and V. M. Shalaev, "Superlens based on metal-dielectric composites," Physical Review B, vol. 72, no. 19, Article ID 193101, 4 pages, 2005.

[25] P. A. Belov, R. Marqués, S. I. Maslovski et al., "Strong spatial dispersion in wire media in the very large wavelength limit," Physical Review B, vol. 67, no. 11, Article ID 113103, 4 pages, 2003.

[26] J. Elser, R. Wangberg, V. A. Podolskiy, and E. E. Narimanov, "Nanowire metamaterials with extreme optical anisotropy," Applied Physics Letters, vol. 89, no. 26, Article ID 261102, 3 pages, 2006. 

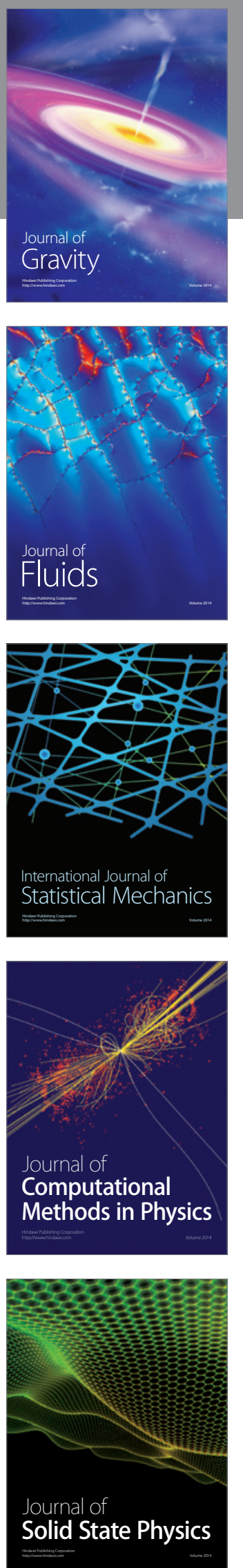

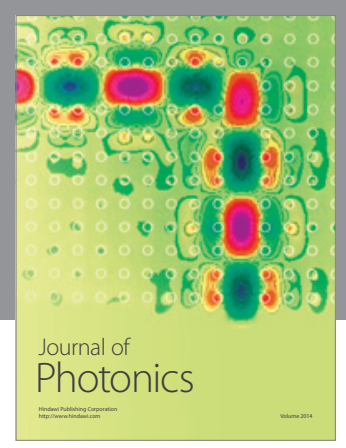

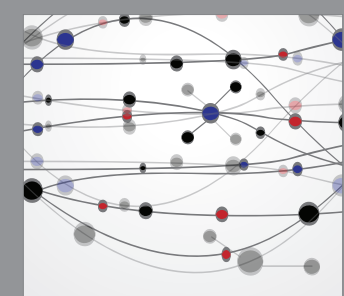

The Scientific World Journal
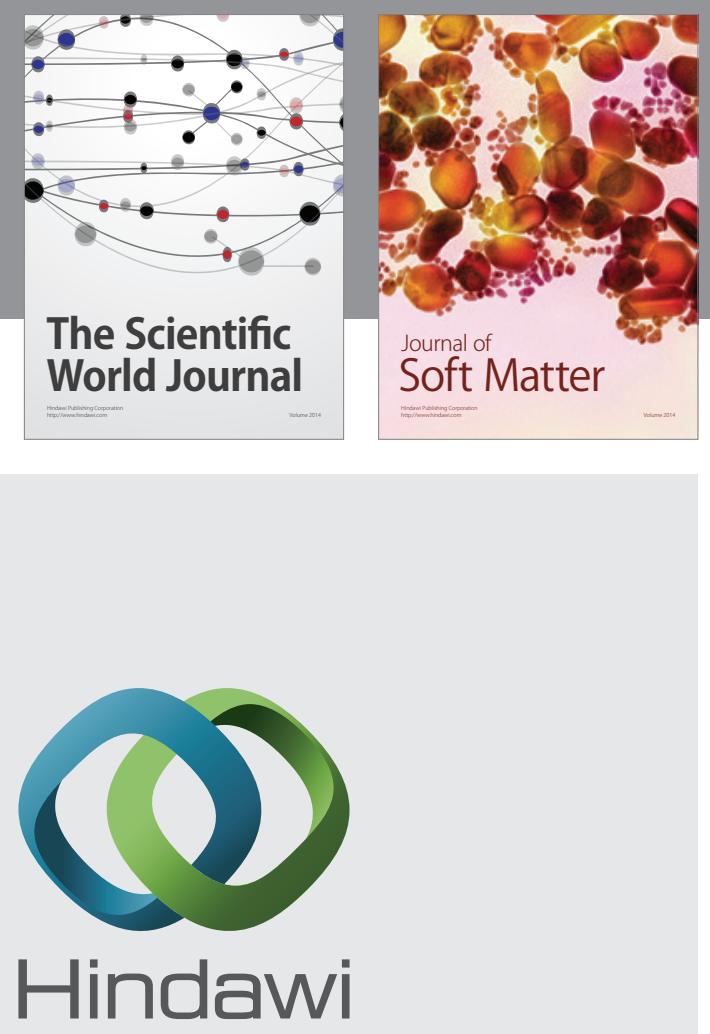

Submit your manuscripts at

http://www.hindawi.com
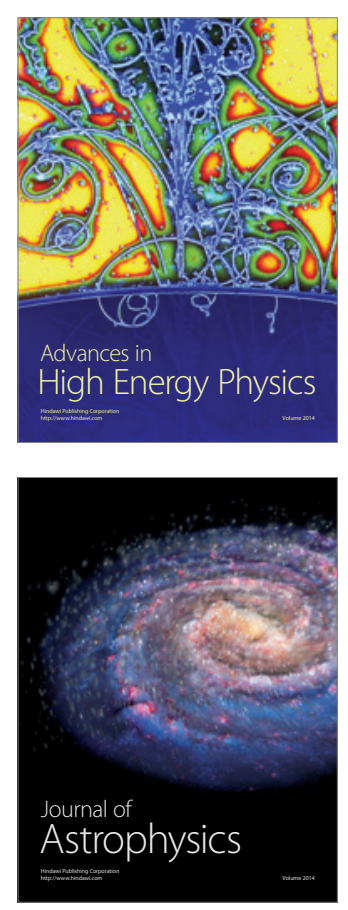
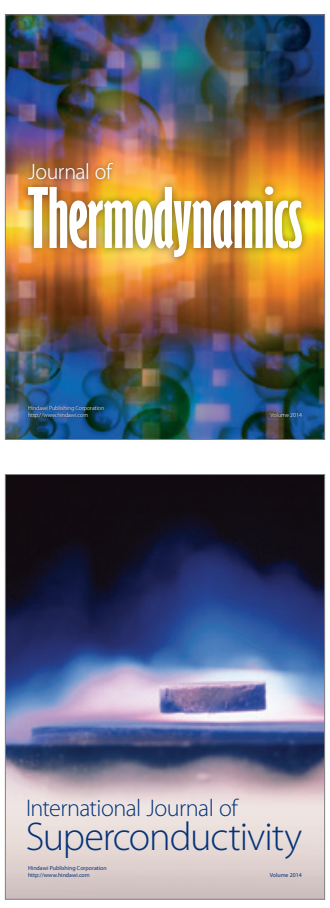
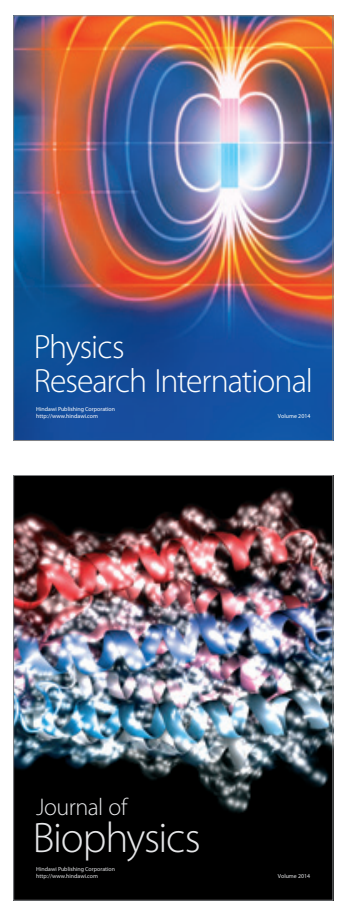
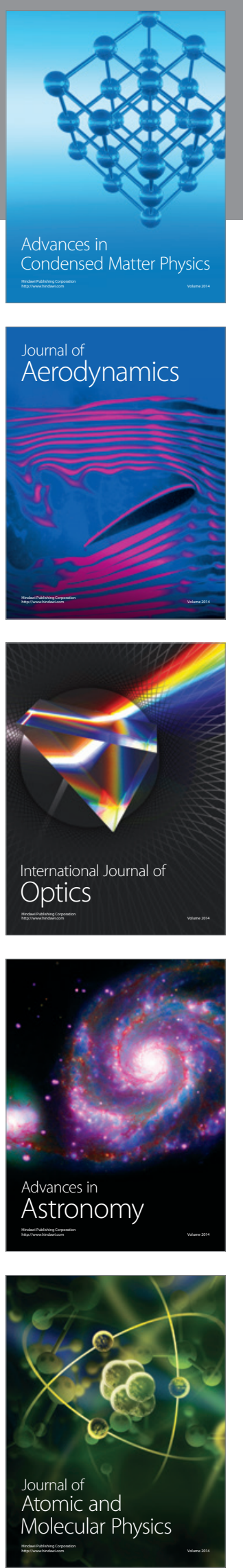